\section{The Preparation and Purification of Perhydrocoronene for Use as a Spectroscopic Matrix}

\author{
John C. Fetzer and W. R. Biggs \\ Chevron Research and Technology Company, \\ Richmond, CA, USA \\ Maximilian Zander \\ Rütgerswerke AG, Castrop-Rauxel, FRG \\ Z. Naturforsch. 46a, 291-292 (1991); \\ received December 10, 1990
}

Perhydrocoronene has recently been studied as a matrix in low and high temperature luminescence spectroscopy of aromatic compounds. Perhydrocoronene has been found to be a by-product formed during the catalytic hydrocracking of a petroleum feedstock. The isolation and purification of spectroscopy grade perhydrocoronene is described in detail.

Perhydrocoronene (PHC) is a seven-ring saturated hydrocarbon that is essentially flat (the structure is shown in Figure 1). We recently have used PHC as a matrix for fluorescence and phosphorescence spectroscopy $[1,2]$. This was a continuation of a much earlier research program $[3,4]$.

The PHC used in these studies was produced as a by-product during the catalytic hydrocracking of a petroleum feedstock [5]. A large deposit (more than $100 \mathrm{~kg}$ of this material was found) precipitated onto the walls of piping associated with the effluent cooling system of a commercial recycle hydrocracking reactor. It is well known that these reactors produce polycyclic aromatic hydrocarbons, including coronene, during their operation.

This crude material was of a very pale greenish or yellowish white colour, consisted of spherical particles of about $1 \mathrm{~mm}$ diameter and had an extremely fluffy consistency. The apparent density, found by filling a beaker and determining the weight of particles added, was about $0.2 \mathrm{~g} / \mathrm{cm}^{3}$. When the density was determined from dried material that had been dissolved in cyclohexane, it was found to be $1.21 \mathrm{~g} / \mathrm{cm}^{3}$.

By gas chromatography/mass spectrometry it was found that the material consisted to about $99.5 \%$ of perhydrocoronenes. The composition was determined to be $93.8 \%$ perhydrocoronene, $1.2 \%$ methylper-

Reprint requests to Dr. John C. Fetzer, Chevron Research and Technology Company, 100 Chevron Way, P.O. Box 1627, Richmond, California 94802-0827, USA. hydrocoronene, $4.6 \%$ perhydrobenzo [bc]coronene (an eight-ring saturated hydrocarbon), and $0.4 \%$ polycyclic aromatic hydrocarbons (PAHs). The PAHs were determined by reversed-phase high-performance liquid chromatography with diode-array UV absorbance detection to be almost totally benzo[ghi]perylene, coronene, or their partially hydrogenated analogues. Petroleum hydrocracking produces large amounts of coronene, and the hydrogenating conditions are severe enough to totally hydrogenate this PAH. It was rather surprising, however, to find such large amounts of a single compound in such high purity in a commercial facility.

Adsorption chromatography in a $1.25 \mathrm{~m}$ long and $2.5 \mathrm{~cm}$ I.D. glass column was used to purify this crude material. Initially the bed consisted of $1 \mathrm{~m}$ of Woelm activity grade 1 neutral alumina. Subsequently it was found that a bed of $0.5 \mathrm{~m}$ of Biorad AG-4 basic alumina and $0.5 \mathrm{~m}$ of Biorad AG-10 acidic alumina performed much better. Particle size for both of these adsorbents was 100-200 mesh. The packings were heated overnight in a vacuum oven (with a pressure of $325 \mathrm{~Pa}$ ) at $110{ }^{\circ} \mathrm{C}$ to remove any adsorbed water. The eluting solvent was liquid-chromatography grade cyclohexane from Burdick and Jackson Laboratories. The purity of the chromatographic fractions was determined by UV absorbance spectrometry. Literature values of molar absorptivities were used $[6,7]$. The chromatographic bed consisting of both acidic and basic alumina was found to selectively remove aromatic molecules from saturated ones [8]. This finding arose from earlier work on selective removal of nitrogen compounds from hydrocarbon material [9].

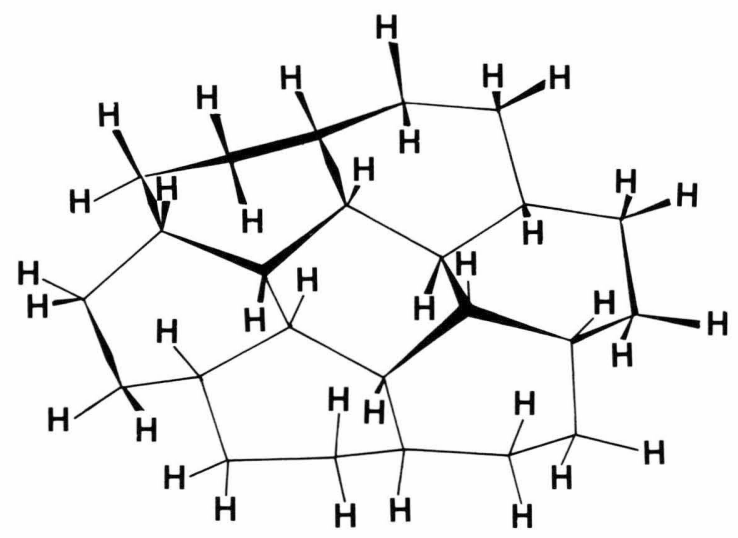

Fig. 1. Structure of perhydrocoronene. 
Vacuum sublimation was used to further purify the PHC. The system consisted of a tube furnace capable of heating the samples to temperatures from 200 to $1000{ }^{\circ} \mathrm{C}$, and a pumping system. The pumping system had a mechanical rotary pump that could produce a vacuum of $25 \mathrm{mPa}$ and a diffusion pump that could reach pressures as low as $15 \mu \mathrm{Pa}$. Because of its relative volatility, PHC required that the oven was set at the minimum temperature, and only the forepump of the vacuum system needed to be used. Quartz sample

[1] J. C. Fetzer and M. Zander, Z. Naturforsch. 45a, 727 (1990).

[2] J. C. Fetzer and M. Zander, Z. Naturforsch. 45a, 814 (1990) (erratum ibid. 45a, 1218 (1990)).

[3] M. Zander, Naturwiss. 44, 443 (1960).

[4] M. Zander, Z. Naturforsch. 29a, 1520 (1974)

[5] R. F. Sullivan, M. M. Boduszynski, and J. C. Fetzer, Energy and Fuels 3, 603 (1989). tubes, approximately $45 \mathrm{~cm}$ long and $1 \mathrm{~cm}$ I.D. with a $20 \mathrm{~cm}^{3}$ cylindrical bulb at one end and a vacuum glass joint at the other, were used.

Sublimation resulted in deposition of a film of PHC on the tube walls. The purity could be visually estimated because the PHC formed a compact matrix. If any coronene were also carried over (because the sublimation rate was too high), the PHC emitted a yellow phosphorescence of long lifetime when illuminated with UV light, identical to an effect previously seen [3].

[6] Robert A. Friedel, Ultraviolet Spectra of Aromatic Compounds, University Microfilms, Ann Arbor, Michigan, USA (1964).

[7] H. Fromherz, L. Thaler, and G. Wolf, Z. Elektrochem. 49, 387 (1943).

[8] J. C. Fetzer, Org. Prep. Proced. Intern. 20, 223 (1988).

[9] M. M. Boduszynski, Energy and Fuels 2, 597 (1988). 